\title{
Number of Classes of Invariant Equilibrium States in Complex Thermodynamic Systems
}

\author{
Jan Turulski \\ Department of Chemistry, Third Age University (TAU), Czarna Wies Koscielna, Poland \\ Email address: \\ jan.turulski@protonmail.com, turul@uwb.edu.pl \\ To cite this article: \\ Jan Turulski. Number of Classes of Invariant Equilibrium States in Complex Thermodynamic Systems. American Journal of Physical \\ Chemistry. Vol. 8, No. 1, 2019, pp. 17-25. doi: 10.11648/j.ajpc.20190801.13
}

Received: April 5, 2019; Accepted: May 14, 2019; Published: June 11, 2019

\begin{abstract}
The values of the Gibbs function of a system with $C$ components create a 2-dimensional topological manifold that is piecewise smooth and continuous. Each of the $C+2$ smooth elements of such a manifold represents the states of a phase within the system. The elements are glued together along the $C$ types of phase transformation lines, which converge to a single point that represents the invariant state of the system (i.e. a state with zero degrees of freedom). Transformation lines, treated as edges, and the smooth elements of the manifold, i.e. faces, constitute a zero-vertex graph that represents the invariant state. This graph is referred to here as the graph-map of the invariant state. The distribution of each component in an invariant state depends on the configuration (distribution) of the phase transformation lines. Because the smoothness and continuity of the manifold makes certain configurations of the lines forbidden, some forms of invariant states are also forbidden, even though they satisfy the Gibbs phase rule. Some academic handbooks do not take this fact into account, and provide forbidden configurations as examples of invariant states. States that only differ in terms of the permutation of two or more of their components will belong to the same class. This study shows that all real graph-maps can be represented by $C$-vertex graphs with $C+2$ edges that have an even value of the vertex valence. The number of such graphs, i.e. the number of classes of invariant states, $\eta_{\mathrm{o}}(C)$, is shown to meet the recurrence relation $\eta_{\mathrm{o}}(2 k+1)=2 * \eta_{\mathrm{o}}(2 k)-\eta_{\mathrm{o}}(2 k-1)$, where $k=1,2,3,4$. Knowing the number $\eta_{\mathrm{o}}(C)$ for several small values of $C$ allows us to determine the number of invariant states in a thermodynamic system using the above equation, regardless of the complexity of the system.
\end{abstract}

Keywords: Graph Theory, Thermodynamic Equilibrium, Invariant Thermodynamic State

\section{Introduction}

It was shown how by using the graph representation of equilibrium states, described in the previous work, determine the qualitative composition of all thermodynamically permissible phases of invariant states in complex systems. The dependence of the number of classes of such states on the number of components of the system was found. It was shown which invariant states are forbidden in thermodynamics. First things first.

Papers trying to apply the theory of graphs in thermodynamics [1-10] date from almost a century. It is worth noting, however, that in these publications the authors did not undertake the investigation of invariant states in complex thermodynamic systems. The presented work is therefore the first and only work on the subject determining the number of invariant states and the composition of individual phases in complex thermodynamic systems. The multiphase chemical system is in thermodynamic equilibrium if the chemical potentials of the individual components in all phases are equal. This condition leads to the Gibbs phase rule binding the number of parameters describing the equilibrium (number of degrees of freedom) $f$, with the number of components $C$, and the number of phases $P$, of the system. As a general rule, when $P=C+2$, the equilibrium describes zero thermodynamic parameters: $f=0$. It means that such a state can exist only at one point, with strictly defined thermodynamic parameters. Changing any parameter in this state disturbs the balance, causing a transition to a state with fewer phases. Therefore, the state with the maximum number of phases $(P=C+2)$ is called the invariant state (state with zero degrees of freedom). Until recently, invariant states have been studied only in one-component systems. Investigation of such a state was rather a simple task, as it was about 
establishing the value of pressure and temperature at which three phases can coexist at the same time. In complex systems, starting from binary ones, the invariant state was in thermodynamics a complete "terra incognita". This state is realized by a specific distribution of $C$ components between $C+2$ phases.

From the combinatorial point of view, there can be many such distributions. Theoretically, therefore, there can be many classes of invariant states in complex systems. Until recently, thermodynamics could not answer whether there is only one type of invariant state in chemistry (which?) among the whole lot of combinatorially of permitted ways of distributing $C$ components between $C+2$ phases. Or maybe thermodynamically will be allowed several (which, how many?) types / classes of invariant state?

The lack of answers to these questions caused that the researchers gave examples of invariant states in complex systems guided by intuition, which, as will be shown in this work, is sometimes unreliable. Partial answer to the above questions was provided by the first work in this series [10]. In it, you can find a more reliable tool than the intuition necessary in the study of invariant states. I am sending this interested reader to this work.

As shown in [10], each thermodynamic equilibrium state represents a planar graph on the topological manifold of the Gibbs function (topological manifold of the function $=$ the hypersurface created by the set of points representing the values of the function) [10]. The vertices of this graph represent degrees of freedom; edges - individual components (exactly, chemical potentials of individual components); faces - individual phases of the system (strictly a set of states of these phases).

In this way, in the work the concept of a zero-vertex graph appeared, which represents the invariant state of the system, [10]. The graph ( $C$ edge and $C+2$ faces) is nothing but a map of the area around the invariant point on the topological manifold of Gibbs' function. Since the Gibbs function is a continuous division such should not lead to the appearance of holes in the surface. In this way, the problem of thermodynamically allowed invariant states has been reduced to the number of non-isomorphic maps on which the $C$ edges divide the surfaces of the manifold into $C+2$ pieces. Divide so that the division does not "hole" the surface.

It can be easily seen that in this way the problem of finding invariances became a trivial problem. In the abovementioned work [10] it was shown that in binary systems there can be three, and in ternary systems - five, different classes of invariant state. For systems with more than 3 components, the problem of finding allowed invariant states by finding a graph - map is still a trivial matter, but unfortunately very laborious and laborious. This last defect results from the inconvenience of operating with zero-vertex graphs. Therefore, in the present work an alternative way of representing invariant states has been proposed. This method is a consequence of the observation that inconvenient at work zero-vertex map graphs are isomorphic with ordinary / normal graphs. Isomorphism is defined as follows: edge graph - map $\leftrightarrow$ vertex of the normal graph

face graph - map $\leftrightarrow$ edge of a normal graph

According to the above mentioned isomorphism, the invariant state in the $C$ component systems taking into account the fact that the graph - map lies on the surface without holes, shows that the normal graphs resulting from this isomorphism will have $C+2$ edges and an even valence (This graph property is also called the "vertex degree". Since one word "degree" in this work occurs in the thermodynamic context ("degree of freedom"), I will continue to use the term "valence of the vertex".) of all $C$ vertices. Because constructing such graphs is extremely easy (in the presented work such graphs for $C<9$ are shown) and thus finding the allowed invariant states in complex systems becomes a solved problem. The solution to this problem has also shown that in the textbooks [11], as an example of an invariant state in binary systems, it is forbidden from the thermodynamic point of view, because its state would have to represent Gibbs' "full of holes" function. While counting individual graphs of the invariant state, it was noticed that the number of these states is related to a simple recursive relation that allows calculation of the total number of invariant states classes in arbitrarily complex systems.

The results presented in the work allow to formulate answers to the above-mentioned questions:

i. in complex $C$ component systems, there may be not one but many classes of invariant states;

ii. the number of these classes can be calculated on the basis of the recurrence dependence presented in the paper;

iii.each class of invariant states is represented by a graph with $C+2$ edges and $C$ evenly valence vertices;

iv. the composition of all the phases of the invariant state is easily determined on the basis of an invariant state graph.

At the end, I realize that at the present moment the problem of determining the form and number of invariant states in very complex systems, rather has no practical significance, and serves only to satisfy the curiosity of a chemist. Will it always be that way?

\section{Theory}

The thermodynamic state in a isothermal-isobaric system is described by the values of the pressure, $p$, temperature, $T$, and the chemical composition, $x_{1}, x_{2}, \cdots, \widetilde{x}_{C}$, where $x_{\mathrm{i}}$ is the mole fraction of the $i$-th component (the tilde over the symbol of one of the fractions means that for closed systems, the fraction is determined by the values of the other fractions: $\left.\tilde{x}_{C}=1-\left(x_{1}+x_{2}+\cdots+x_{C-1}\right)\right)$ where the values are the arguments of the Gibbs function $G\left(p, T, x_{1}, x_{2}, \cdots, \tilde{x}_{C}\right)$. In such conditions, equilibrium is defined by the minimum of the Gibbs function. This minimum enforces equality on the chemical potentials, $\mu_{i}^{(n)}$, of each of the $C$ components, $i=$ A, B, C, ... in each of the $P$ phases of the system: 


$$
\begin{aligned}
& \mu_{i}^{(n)}\left(p, T, x_{1}^{(n)}, x_{1}^{(n)} x_{2}, \cdots \cdot, \tilde{x}_{C}^{(n)}\right)=\mu_{i}^{(m)}\left(p, T, x_{1}^{(m)}{ }_{1}, x_{1}^{(m)} x_{2}, \cdots \cdots, \tilde{x}_{C}^{(m)}\right) \cdots \\
& n \neq m=\alpha, \beta, \gamma, \ldots ; \cdots i=A, B, C, \ldots
\end{aligned}
$$

where:

$$
\mu_{i}^{(n)}=\left(\frac{\partial \Delta G^{(n)}}{\partial x_{i}}\right)_{p, T, x_{j}}
$$

The equations (1) cause the number of variables that characterize the equilibrium to decrease from $P^{*}(C-1)+2$ to what is referred to as the number of degrees of freedom in a system, $f$ :

$$
f=C-P+2
$$

It is easy to notice that the number of variable parameters, $f$, that characterize the equilibrium decreases with the number of phases of the system, $P$. If $P$ is equal to $C+2$, the equilibrium becomes invariant, i.e. equilibrium can only exist in a single, particular state; whereas altering any of the thermodynamic parameters of this state will break the invariance. However, it would be incorrect to believe that satisfying the equation $P=$ $C+2$ under the strictly defined values of the thermodynamic parameters is sufficient to make a system invariant, as this is only true for unary systems. In complex systems, the sufficient conditions for invariance result from the fact that the point representing the invariant state is the intersection point for the phase transformation lines located on the Gibbs function topological manifold [10].

In order to explain the nature of the sufficient conditions, we should first outline the topological interpretation of thermodynamic equilibria [10]. The topological equivalent of the Gibbs phase rule (3) is referred to as Euler's theorem for planar graphs on 2-D surfaces isomorphic to the surface of a sphere [12]. If $f$ denotes the number of vertices of a planar graph, $C$ denotes the number of edges, and $P$ denotes the number of faces, then according to Euler's theorem, the parameters of the graph are given by Equation (3), i.e. an equation that is identical to the Gibbs phase rule. As Study [10] shows, this correspondence between the theorems from two different fields of science allows us to propose that the values of the Gibbs potential create a 2-D, piecewise smooth and continuous (i.e. with no holes), topological manifold. The above study proves that, by applying the conditions that result from Gibbs-Duhem's theorem onto a function, the dimensions of the manifold of that function $G\left(p, T, x_{1}, x_{2}, \cdots, \widetilde{x}_{C}\right)$ are reduced (from $C+1$ to 2 ) [10]. The same study shows that the state of thermodynamic equilibrium is represented by planar graphs located on the manifold, which are referred to as state graphs [10]. The correspondence between the phase rule and Euler's theorem has been known in the field of Chemistry since the 1930s [10]. However, researchers were unable to recognize this correspondence for a long time. One reason, though not the only one, for this inability was an issue concerning the state graphs for systems in an invariant state. As has already been explained, such graphs should not have any vertices $(f=0)$ and should contain $C$ edges and $C+2$ faces. Consequently, vertex-less graphs were considered to be an impossibility. Some researchers believed that the correspondence between the phase rule and Euler's theorem was coincidental (after all, how could an invariant state possibly be represented with a vertex-less graph!). However, Study [10] shows that such a graph is simply comprised of two phase transformation lines located on the Gibbs function topological manifold. These lines constitute the edges of the graph of the invariant state, and its faces are the smooth faces of the manifold, which correspond to the states of each phase within the system. Figure 1 presents such a graph for a unary system, $C=1$. As can be seen in the figure, the graph constitutes a mapping of the topological manifold onto the plane $(p, T)$. In other words, the graph is a 2-D map of the manifold, which is why we can propose calling such a graph a graph-map. The faces of the graph are marked with Greek letters, while the segments of the edges are marked with Latin letters: $k, l, m \ldots$

We use the term segments of the edges, rather than edges, because the graph does, in fact, contain a single edge that bifurcates at the invariant point. Why a single edge instead of three? Firstly, because $f=0$ and $C=1$ only enable a single edge to be defined by Equation (1). If we denote the components of the system as $\mathrm{A}, \mathrm{B}, \mathrm{C} \ldots$ and assume that the unary system contains the component A, then Equation (1) will take the following form:

$$
\left\{\begin{array}{l}
k: \cdots \cdots \cdots \mu_{A}^{\alpha}=\mu_{A}^{\beta} \\
l: \cdots \cdots \cdots \mu_{A}^{\beta}=\mu_{A}^{\gamma} \\
m: \cdots \cdots \cdot \mu_{A}^{\gamma}=\mu_{A}^{\alpha}
\end{array}\right\}
$$

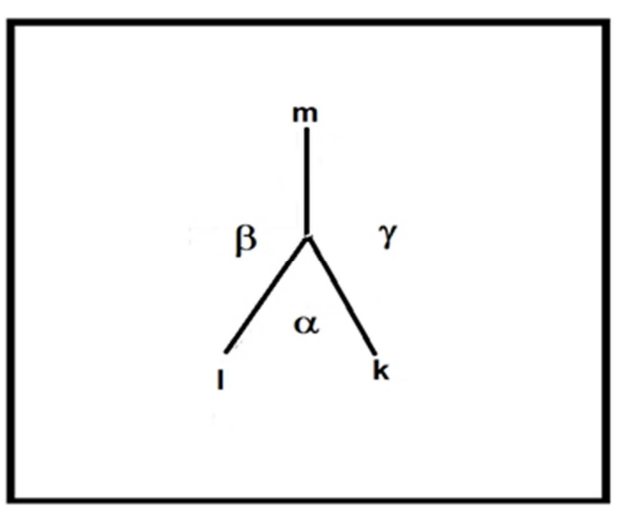

Figure 1. Mapping the Gibbs function topological manifold for a unary system onto the plane $(p, T)$ creates a graph-map of an invariant state. The edge of the graph-map, i.e. the line $k, l, m$ that bifurcates in the invariant state, is given by Equations (4) and is comprised of segments $\alpha, \beta, \gamma$ which determine the equilibrium of each phase.

That is, the edge in the graph-map is given by the equations that define the equilibrium of the individual components within the corresponding phases of the system. Let us explain this using the invariant states in binary systems, $C=2$, as an example. To differentiate between the 
components, we will denote them on the graph-map as: $\mathrm{A}-\mathrm{a}$ continuous line; $\mathrm{B}-\mathrm{a}$ dashed line; $\mathrm{C}-\mathrm{a}$ dotted line, etc. Figure 2 shows the graph-maps for all the invariant states that are possible in binary systems. As the figure indicates, three invariant states exist in binary systems. Both edges (even though they are composed of several segments) of the graphs in these states will now be defined, respectively, by:

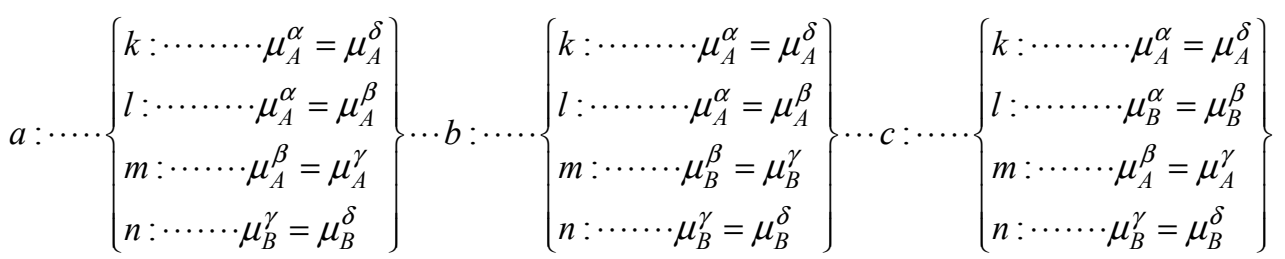

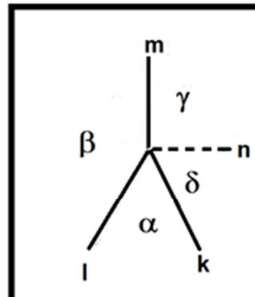

a

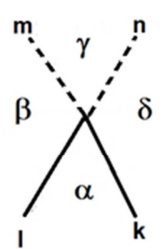

b

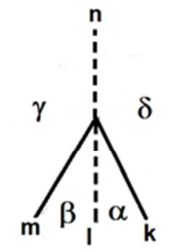

c
Figure 2. Mapping the Gibbs function topological manifold for a binary system onto the plane $(p, T)$ creates a graph-map of three invariant states that are possible in the binary states $(a, b, c)$. The edges of the graph-map, i.e. the lines $k, l, m, n$ that bifurcate in an invariant state, are given by Equations (5) and are composed of segments $\alpha, \beta, \gamma, \delta$ which determine the equilibrium of each phase. Component $A$ (specifically, the chemical potential of Component A) is marked in the graph with a continuous line, and Component $B$ is marked with a dashed line. Phase compositions in figures a, $b$, c give Eq.(6).

Note that each edge segment of a given component is associated with two different phases, $X$ and $Y$ (even though the compositions of the phases cannot differ qualitatively). As a result, the edge of any component, $i=\mathrm{A}, \mathrm{B}, \mathrm{C} \ldots$, will be associated with an even number of phases in total. Unfortunately, using such graph-maps to represent the states of complex systems is inconvenient, and equations such as (5) create lengthy descriptions of these graph-maps; hence there is the need for an alternative method of encoding each invariant state in these complex systems. Note that every graph of a complex system is comprised of faces that represent the state of each phase. Every face is separated on two sides by an edge segment, i.e. a line that is defined by the chemical potential of the components $\mathrm{X}$ and $\mathrm{Y}$, respectively. It will be convenient to denote such a face representing a particular phase by enclosing the symbols of these components in parentheses, as in $(\mathrm{X} . . \mathrm{Y})$. If face $(\mathrm{X} . . \mathrm{Y})$ corresponds to several phases, it will also be reasonable to precede its symbol with the number denoting its multiplicity, instead of repeating the symbol of the face multiple times. However, if both components are the same, we will simply use (X) instead of (X... X). All the faces encoded in this manner will be enclosed in square brackets. The graph-maps of the invariant states in Figure 2 will then be encoded with equations composed of the following sequence of symbols:

$$
a: \cdots \cdots[2(A) 2(A \ldots B)] \cdots \cdots b: \cdots \cdots[(A)(B) 2(A \ldots B)] \cdots \cdots c: \cdots \cdots[4(A \ldots B)]
$$

These equations allow for the creation of new graphs (different to the graph-maps), which will prove useful for counting the invariant states. The vertices of such graphs denote the individual components, A, B, C..., of the system (or, strictly speaking, the chemical potentials of the components), while their edges (including the loops) represent the phases and indicate what components of the system that are present in a given phase. For instance, a graph corresponding to Formula " $a$ " in Equation (6) will have two vertices connected with two edges, with vertex $\mathrm{A}$ also containing two loops. At this point [12] it is useful to remember that the valence of a vertex is given by the total number of edges coming into and out of the vertex (which means that a loop contributes twice as much to the valence!). This information, together with the above information describing the number of phases that are associated by the edges of a given component on a graph-map, leads to the conclusion that the graphs of invariant states will have vertices with an even value of valence. Figure 3 presents three graphs that were created in this manner for binary systems, while the corresponding maps are shown in Figure 2.

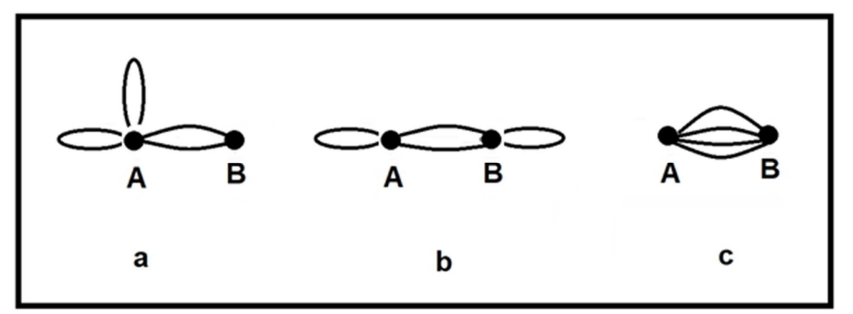

Figure 3. A different graph representation of the invariant states in a binary system. Each graph corresponds exclusively to a particular graph-map from Figure 2. The vertices with an even numbered valence represent the individual components of the system A, B (specifically, the chemical potentials of these components), and the edges of the graphs are the individual phases $\alpha, \beta, \gamma, \delta$ of the system.

At this point, the following question arises: "Do such graphs of invariant states account for all possible invariant states in a system with $C$ components?" Unfortunately, the 
answer is "no". The graph-maps constructed in the aforementioned manner and the graphs of invariant states introduced a moment ago are only representatives of the individual classes of invariant states. It seems that the content of each class can be determined with at least two methods. Let us begin with the simplest method.

1. The first class can be expanded onto each state by exchanging the components of the class representatives, e. g. $\mathrm{A} \leftrightarrow \mathrm{B}$ or $\mathrm{A} \leftrightarrow \mathrm{C}$, etc. Thus, we will obtain all possible graphs/formulas of the invariant states. From this point on, we will separate the names of the components in all the formulas within each phase with commas, rather than an ellipsis (as was the case for the classes), e. g. (A, B). We can observe that for the class of the binary state graphs (6), the exchange $A \leftrightarrow B$ does not create any new invariant states for the classes marked " $b$ " and "c" in Equation (6), but it does create an additional state for the class marked "a". Thus, Equation (6), which describes the classes of the invariant states, is transformed into Equation (7), which describes the individual states within these classes:

$$
a: \cdots \cdots[2(A) 2(A, B)], \cdot \cdot[2(B) 2(A, B)] \cdots \cdot b b: \cdots \cdots[(A)(B) 2(A, B)] \cdots \cdots c: \cdots \cdots[4(A, B)]
$$

In other words, binary systems can come with four different invariant states: two belonging to one class and one belonging to each of the other two classes. We will call the classes that contain more than one invariant state degenerate classes. If an invariant state belongs to a degenerate class, then it is impossible to determine which state of this class in particular constitutes that invariant state, e. g. whether the state in question is $[2(\mathrm{~A}) 2(\mathrm{~A}, \mathrm{~B})]$ or $[2(B) 2(A, B)]$. This is because every thermodynamic system can contain only a single invariant state (we treat each allotrope and polymorph as different substances), and thus, we do not know which permutation of the components will occur in a given state. Consequently, it seems reasonable to use the classes of states, rather than the states themselves, to count the invariant states.

A graph created for an invariant state for a particular thermodynamic system should belong to a class of graphs. If it does not, then we can conclude with full certainty that such a state does not exist in reality. It is worth bearing this information in mind, even though some authors of academic handbooks [11] provide a forbidden equilibrium as an example of an invariant equilibrium in a complex system $(C$ $=2$ ). Specifically, Karapientiantz [11] claims with respect to a water-salt system (let us denote these components as A and $\mathrm{B}$, respectively) that the invariant equilibrium corresponding to this system contains a saturated solution of salt (phase $\alpha$ ), ice (phase $\beta$ ), steam $(\gamma)$, and salt crystal sediment $(\delta)$. Such an example of an invariant state seems so natural that it is astonishing to learn that the state is impossible in reality! Why? Let us consider the formula that encodes this state: $[2(\mathrm{~A})(\mathrm{B})(\mathrm{A}, \mathrm{B})]$. The list of binary invariant states (7) does not include a state with this formula. However, that does not yet prove the nonexistence of such a state. Therefore, let us consider the graph-map and the state graph that are shown in Figures $4 \mathrm{a}$ and $4 \mathrm{~b}$. The graph-map of this state (Figure $4 \mathrm{a}$ ) indicates the presence of a hole in the Gibbs function topological manifold (the hole is marked with a question mark in the figure). The Gibbs function of this state would have to be discontinuous, which is impossible.

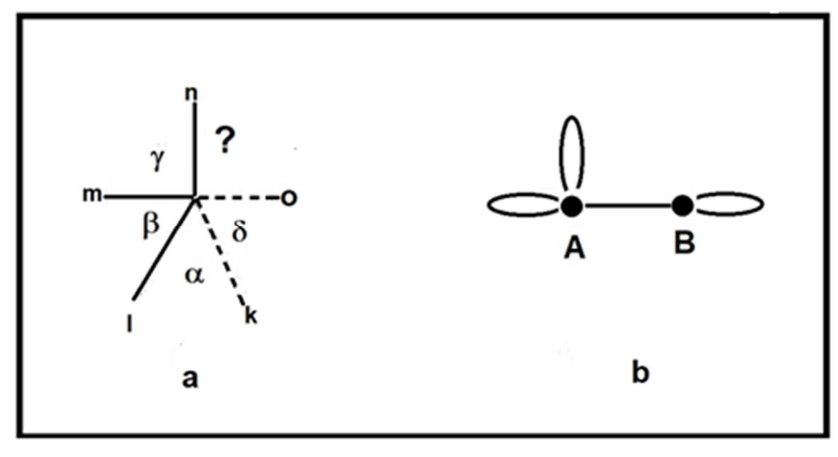

Figure 4. A graph-map (Figure 4a) and its corresponding graph (Figure 4b) of an invariant state in a binary system, which [3] claims occurs in a watersalt system. In actuality, the hole in the graph-map (the area marked with "?" in Figure 4a) indicates that such a state does not exist due to a discontinuity of the Gibbs function manifold. The non-physicality of the state is also demonstrated by its graphical representation (Figure 4b), as the vertices of the graph have an odd-numbered valence.

Thus, we must accept the fact that the invariant state of the water-salt system proposed in [3] does not exist! Furthermore, it does not exist even despite the apparent soundness of the proposed phases and their correct number, as that "sound" phase composition leads to a discontinuous Gibbs function. At this point, let us add that in the correct invariant state, either phase $\beta$ or $\delta$ (but not both) will be binary, rather than unary, as the author [11] suggests.

Furthermore, we can consider the state graph in Figure $4 \mathrm{~b}$ that corresponds to such a discontinuous graph-map. It is easy to notice that the valence of at least some of the vertices of the graph is odd-numbered (or even all of the vertices, as in this case). An odd value of the valence is characteristic for the graphs that correspond to discontinuous graph-maps, which include not only $[2(\mathrm{~A})(\mathrm{B})(\mathrm{A}, \mathrm{B})]$, but also $[3(\mathrm{~A})(\mathrm{A}, \mathrm{B})]$, $[(\mathrm{A}) 3(\mathrm{~A}, \mathrm{~B})], \quad[(\mathrm{A})(\mathrm{B})(\mathrm{C})(\mathrm{A}, \mathrm{C})(\mathrm{B}, \mathrm{C})]$, etc. Therefore, invariant states are represented by an even-numbered valence of vertices, with the number of vertices equal to the number of components, $C$, and the number of edges equal to the number of phases of the invariant state, $C+2$. The Appendix to this study provides all such graphs for systems containing no more than 6 components. By counting the graphs for each $C$, we will arrive at their numbers, marked $\eta_{\mathrm{o}}$, which are equal to the number of classes of the invariant states. Table 1 shows the values of $\eta_{\mathrm{o}}$ obtained in this manner, including the 
values of $\eta_{\mathrm{o}}$ for $C \leq 8$ (the graphs for $C=7$ and $C=8$ are excluded from the Appendix due to their high number). The table indicates that, for each system with an even number of components, its $\eta_{\mathrm{o}}$ differs by the same amount from the $\eta_{\mathrm{o}}$ of both its neighbors with an odd number of components. In Table 1, these numbers are 2, 2, 5, 5, 9, 9, 14, and 14 .

Table 1. Number of graphs of invariant state classes, $\eta_{o}$, for systems with no more than 8 components $(C \leq 8)$. The parameter $\Delta=\eta_{o}(C)-\eta_{o}(C-1)$ indicates that the $\eta_{o}$ for states with an even number of components differs by the same amount from both of its neighbors with an odd number of components.

\begin{tabular}{|c|c|c|c|c|c|c|c|c|c|c|c|}
\hline k & & 1 & & 2 & & 3 & & 4 & $\ldots$ & $\ldots$ & $\mathbf{k}$ \\
\hline $\mathrm{C}$ & 1 & 2 & 3 & 4 & 5 & 6 & 7 & 8 & $\ldots$ & $\ldots$ & $2 \mathrm{k}$ \\
\hline$\eta_{\mathrm{o}}$ & 1 & 3 & 5 & 10 & 15 & 24 & 33 & 47 & $\ldots$ & $\ldots$ & \\
\hline$\Delta$ & & 2 & 2 & 5 & 5 & 9 & 9 & 14 & $\ldots$ & $\ldots$ & $\mathrm{k}(\mathrm{k}+3) / 2$ \\
\hline
\end{tabular}

The application of the table difference method [13, 14] for this sequence of integers indicates that the above difference for any $C=2 k$, where $k=1,2,3,4, \ldots$ will be given by:

$\eta_{\mathrm{o}}(2 k)-\eta_{\mathrm{o}}(2 k-1)=\eta_{\mathrm{o}}(2 k+1)-\eta_{\mathrm{o}}(2 k)=k(k+3) / 2$, where $k=1$,

$$
2,3,4 \ldots
$$

Note that Equation (8) leads to the conclusion that the $\eta_{\mathrm{o}}$ for systems with an even number of components meet the following equation:

$$
\eta_{\mathrm{o}}(2 k+2)=\eta_{\mathrm{o}}(2 k)+\left(k^{2}+4 k+2\right), \text { where } k=1,2,3,4,
$$

Equations (8) and (9) can then be easily transformed into the following second-degree recurrence relation for $\eta_{\mathrm{o}}$ :

$$
\eta_{\mathrm{o}}(2 k+1)=2 * \eta_{\mathrm{o}}(2 k)-\eta_{\mathrm{o}}(2 k-1)
$$

By creating the graphs of the invariant states for several small values of $C$, these equations can be used to calculate the $\eta_{\mathrm{o}}$ for thermodynamic systems of any complexity ${ }^{1}$. Of course, these numbers will indicate the number of classes of invariant states, rather the number of the states themselves. The latter number can be obtained by following the procedure described in Section 1, i.e. by permutations the components. However, there is another method for expanding the classes into a set of states, which we will now describe based on a particular example. Consider Graph 3 for $C=3$ in the Appendix. Let us assume that the vertices of the graph represent the individual components $\mathrm{A}, \mathrm{B}, \mathrm{C} \ldots$ in order of decreasing valence (if multiple vertices have the same valence, then the order is irrelevant). Thus, the graph will represent the class of invariant states that is given by the formula $[3(\mathrm{~A} \ldots \mathrm{B})(\mathrm{A} \ldots \mathrm{C})(\mathrm{C} \ldots \mathrm{B})]$. The method described in Section 1 (permutation of the components) allows for the conclusion that this class represents such states as:

$$
[3(\mathrm{~A}, \mathrm{~B})(\mathrm{A}, \mathrm{C})(\mathrm{B}, \mathrm{C})][3(\mathrm{~A}, \mathrm{C})(\mathrm{A}, \mathrm{B})(\mathrm{B}, \mathrm{C})]
$$

\footnotetext{
${ }^{1}$ It turns out that the simple mathematical transformations of equations (8), (9) and (10) lead to the following explicit dependence of the number of classes of invariant states, $\eta_{\mathrm{o}}$, on the number of independent components: $\eta_{\mathrm{o}}(2 \mathrm{k})=3+2 *(\mathrm{k}-1)(\mathrm{k}+1)+\mathrm{k} *(\mathrm{k}-1)(2 \mathrm{k}-1) / 6$, $\eta_{\mathrm{o}}(2 \mathrm{k}+1)=3+2 *(\mathrm{k}-1)(\mathrm{k}+1)+\mathrm{k} *(\mathrm{k}-1)(2 \mathrm{k}-1) / 6+\mathrm{k} *(\mathrm{k}+3) / 2$, where $\mathrm{k}=1,2,3$, $4, \ldots$
}

$$
[3(\mathrm{~A}, \mathrm{~B})(\mathrm{B}, \mathrm{C})(\mathrm{A}, \mathrm{C})][3(\mathrm{~B}, \mathrm{C})(\mathrm{A}, \mathrm{C})(\mathrm{A}, \mathrm{B})]
$$

As is mentioned above, the states obtained by permutation the components in a given class are uninteresting, and the single invariant state in a system makes these states undifferentiable. Conversely, it would be interesting to determine the maximum number of components in the phases of an invariant state. If we were to mechanically expand the class graphs into each invariant state solely through the permutation of the components, we could conclude that none of the phases of the invariant state could contain more than 2 components, even within the systems with 3 or more components. However, we can disprove this possibility by applying another method for determining the invariant states in a given class, i.e. we will analyze the intersection of 2-D topological manifolds that are immersed in spaces of more than 3 dimensions.

Structures such as planes or smooth 2-D manifolds behave slightly differently in these spaces when compared to their behavior in a 3-D space. Remember that in a 3-D space, two different planes (but also two smooth elements of a 2-D manifold) may either not intersect (they can be parallel to each other) or may intersect only along a straight line. Then, what is the situation for spaces with more than 3 dimensions? Some may even ask, "What use are such spaces for us?" Let us explain. The thermodynamic states of systems constitute points in a space that spans the $C+2$ coordinate axes (pressure, temperature, $C-1$ components, and an axis of the values of the Gibbs function). Specifically, the set of these states forms a 2-D, piecewise smooth manifold immersed in a space with $C+2$ dimensions. We will analyze the behavior of these structures based on the relationship between two ordinary 2-D planes. Let these two planes be immersed in a space that spans the axes marked $\xi_{\mathrm{a}}, \xi_{\mathrm{b}}, \xi_{\mathrm{c}}, \xi_{\mathrm{d}} \ldots$, and let $z$ denote the value axis, as per the usual notation. If the first plane spans the first two axes, and the second plane spans the next two axes, then the two planes are given by:

$$
\begin{aligned}
& z_{\alpha}=a \xi_{a}+b \xi_{b}+m \\
& z_{\beta}=c \xi_{c}+d \xi_{d}+n
\end{aligned}
$$

where $a, b, c, d, m$, and $n$ are the parameters of a plane.

We can then distinguish three cases that show the possible relationships between the planes $z_{\alpha}$ and $z_{\beta}$, which indicate a different method for expanding a class into a set of the individual states.

i. If the axes $\xi_{\mathrm{a}}, \xi_{\mathrm{b}}$ are completely different from $\xi_{\mathrm{c}}, \xi_{\mathrm{d}}$, then both planes either have no common point, because they lie in completely different subspaces; or, if the free parameters are equal, i.e. $m=n$, then the planes have only a single common point, such that $\xi_{\mathrm{a}}=\xi_{\mathrm{b}}=\xi_{\mathrm{c}}=\xi_{\mathrm{d}}$ $=0, z=m$. Of course, in a 3-D space, it is impossible for planes to only intersect at a single point.

ii. If the axes $\xi_{\mathrm{a}}, \xi_{\mathrm{b}}$ are identical to $\xi_{\mathrm{c}}, \xi_{\mathrm{d}}$, then both planes either intersect along the line $\xi_{\mathrm{a}}=\left(-b d^{*} \xi_{\mathrm{b}}-m n\right) / a c$, where $a c=a-c, b d=b-d, m n=m-n$; or, if the parameters are $\mathrm{a}=\mathrm{c}$ and $\mathrm{b}=\mathrm{d}$, then the planes have no 
common points, i.e. they are parallel to each other. These situations occur in a 3-D space.

iii.If two of the axes are identical, e. g. $\xi_{\mathrm{a}}$ and $\xi_{\mathrm{c}}$, but the other two, $\xi_{\mathrm{b}}$ and $\xi_{\mathrm{d}}$, are different, then both planes will also intersect $\left(z_{\alpha}=z_{\beta}\right)$ along a geometric structure given by $\xi_{\mathrm{a}}=\left(-b^{*} \xi_{\mathrm{b}}-d^{*} \xi_{\mathrm{d}}-m n\right) / a c$. This structure is simply a 2-D plane. To summarize, we may state that in spaces with more than 3 dimensions, two planes (but also 2-D topological manifolds) can also intersect at a single point and along a 2-D plane (manifold). The latter case does not occur in an ordinary 3-D space, and it will be of interest to us when we investigate the number of components in each phase of an invariant state.

Another question arises at this point: "What do these topological and geometrical analyses have to do with describing an invariant state?" Invariant states are represented as a point on the phase transformation line, i.e. the line at which two 2-D topological manifolds intersect, with each manifold representing the thermodynamic states of both phases. Of course, each manifold differs from an ordinary plane, but their intersections at spaces with 4 or more dimensions are qualitatively similar to the aforementioned intersection of planes. That is, the manifolds are able to intersect along a line - most likely a curve, rather than on a straight line. Furthermore, the manifolds in spaces with 4 or more dimensions are able to intersect along a 2-D manifold and at a single point. We are interested in their intersections along a 1-D line and a 2-D manifold, and we will analyze the implications of these varied modes of intersection based on a familiar example of a class (10) in a system with $C=3$ given by Graph 3 in the Appendix. Let us now expand the formula for this class to mark the individual phases:

$$
\begin{array}{r}
{[3(\mathrm{~A} \ldots \mathrm{B})(\mathrm{A} \ldots \mathrm{C})(\mathrm{B} \ldots \mathrm{C})] \equiv} \\
{[(\mathrm{A} \ldots \mathrm{B})(\mathrm{B} \ldots \mathrm{A})(\mathrm{A} \ldots \mathrm{B})(\mathrm{B} \ldots \mathrm{C})(\mathrm{C} \ldots \mathrm{A})]}
\end{array}
$$

Let $\alpha, \beta, \gamma, \delta, \phi$ denote the individual phases within this system, as per the usual notation. We will analyze the equilibrium between phases $\beta$ and $\gamma$ of this system as an example. The formula shows that this equilibrium depends on the identicality between the chemical potentials of component $\mathrm{A}$ in both phases:

$$
\mu_{A}^{\beta}=\mu_{A}^{\gamma}
$$

In topological terms, this identicality corresponds to the intersection of the manifold of the chemical potential of component $\mathrm{A}$ in phase $\beta$ and the manifold of the chemical potential of component $A$ in phase $\gamma$. The intersection may sometimes take place along a 1-D line (curve), i.e. a geometrical structure that depends on a single variable, in this case, the content of component A, $x_{\mathrm{A}}$ (see Section 2.2). This type of intersection in the manifolds of both phases does not introduce any additional components into the phases. However, in a space with more than 3 dimensions, Equation (14) may sometimes also describe the intersection in the manifold of the chemical potentials along a 2-D manifold that depends on two variables (see Section 2.3), e. g. the contents of component $\mathrm{A}$ and component $\mathrm{C}$. The latter type of intersection indicates that at least two phases, $\beta$ and $\gamma$, have three components, because their phase transformation "line" depends on the coordinates $x_{\mathrm{A}}$ and $x_{\mathrm{C}}$ :

$$
[(\mathrm{A}, \mathrm{B})(\mathrm{B}, \mathrm{C}, \mathrm{A})(\mathrm{A}, \mathrm{C}, \mathrm{B})(\mathrm{B}, \mathrm{C})(\mathrm{C}, \mathrm{A})]
$$

Thus, the fact that in spaces with more than 3 components, the equilibrium condition (e. g. the one in (14)) may be topologically met in more manners than in a 3-D space indicates that the individual phases of the invariant state may contain more than 2 components. Whether this will be true for particular physicochemical systems is impossible to determine at this point, as the topology of the thermodynamics of the Gibbs function manifold are not welldocumented.

\section{Summary and Conclusion ${ }^{2}$}

To date, invariant states in complex thermodynamic systems have not been the subject of an in-depth theoretical scrutiny [15]. This may be the reason why even some academic handbooks provide incorrect examples of such states, e. g. within binary systems. In our previous study, we showed that the invariant states in systems with $C$ components are represented by a planar graph-map with zero vertices, $C$ edges, and $C+2$ faces. While this representation can be used to count the invariant states, the method is extremely complicated and leads to errors due to the difficulties in establishing the non-isomorphic configurations of the edges in a graph-map, i.e. those that create different graphs. Consequently, in this study an alternative representation has been proposed: an invariant state is represented by a graph with an even-numbered valence, $C$ vertices, and $C+2$ edges. Each such graph corresponds exclusively to a particular graph-map. This representation also has other advantages: it is easy to interpret, i. e . its form allows researchers to easily establish the content of each phase of the state and to easily find all non-isomorphic graphs, even for very complex systems. In the study, it was shown that the number of these graphs, $\eta_{\mathrm{o}}$, and thus, the number of different classes of invariant states for a system with $C$ components, meets the Recurrence Relation (10). This allows $\eta_{\mathrm{o}}$ to be calculated for any value of $C$. Every class can then be expanded into the individual invariant states by permutation its components, although this endeavor is slightly pointless, since the states of a given system are indistinguishable. The classes can also be expanded into the corresponding states by analyzing the intersections between the topological manifolds of each phase. This endeavor is more reasonable, but is also much more difficult due to a lack of theoretical methods for investigating the Gibbs function topological manifold.

\footnotetext{
${ }^{2}$ The paper is dedicated to the memory of my friend Burek, who died on July 21, 2016.
} 


\section{Appendix}

The table included in the Appendix contains the graphs for each class of invariant states for the systems with $C \leq 6$. The graphs for $C=7$ and $C=8$ are so numerous that they have been omitted in the table. Each vertex of a graph corresponds to the individual components of the system composed of $C$ components (specifically, to the chemical potentials of the components), and its edges (including loops) are simply the phases of the system. Numbers on individual state graphs have been placed to inform how many different invariant states exist in $\mathrm{C}$ component systems.

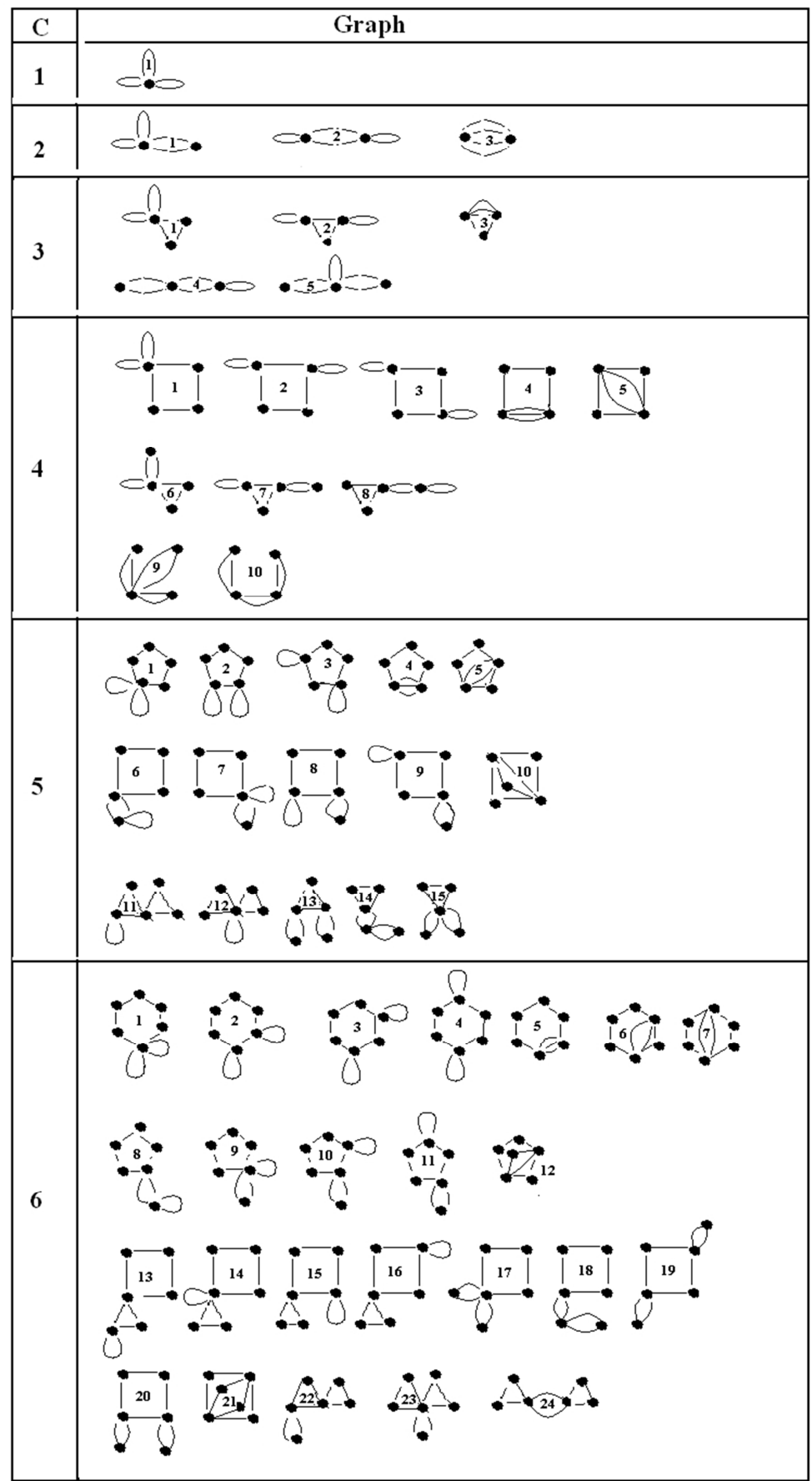

Figure 5. Graphs of all allowed invariant states in thermodynamic systems whose number of independent components is not greater than 6.

[2] M. A. Klochko, Izvest. Sektora Fiz-Khim. Analiza, Inst. Obshch. Neorg. Khim. Akad. Nauk SSSR 19, 82, (1949).

\section{References}

[1] O. Rudel, Z. Electrochem. 35, 54, (1929).
[3] D. R. Rouvray, Chem. Br. 10, 11, (1974).

[4] T. P. Radhakrishnan, J. Math. Chem. 5, 381, (1990). 
[5] L. Pogliani, MATCH Commun. Math. Comput. Chem. 49, 141, (2003).

[6] A. I. Seifer, V. S. Stein, Zh. Neorg. Khim. 6, 2711, (1961).

[7] J. Mindel, J. Chem. Educ. 39, 512, (1962).

[8] F. A. Weinhold, Theoretical Chemistry, Advances and Perspectives, Eds. H. Eyring, D. Henderson, (Academic Press, NY, 1978), vol. 3, p. 15.

[9] J. Turulski, J. Niedzielski, J. Chem. Inf. Comput. Sci. 42, 534, (2002).

[10] J. Turulski, J. Math. Chem. 53, 495, (2015).
[11] M. Ch. Karapientiantz, Chemical Thermodynamics, (Khimia, Moscow, 1975, in Russian).

[12] N. Trinajstic, Chemical Graph Theory, (CRC Press, Boca Raton, 1992).

[13] N. J. A. Sloane N. J. A, S. Plouffe, The Encyclopedia of Integer Sequences, (Academic Press, NY, 1995).

[14] J. H. Conway, R. K. Guy, The Book of Numbers, (Springer Verlag, NY, 1998).

[15] H. DeVoe, Thermodynamics and Chemistry, (2016 web version at: http://www.chem.umd.edu/thermobook), p. 419. 